\title{
Estudo laboratorial do comportamento mecânico de agregados reciclados para aplicação em reaterro de valas
}

\author{
Laboratory study of mechanical behaviour of recycled aggregates for \\ trenches backfilling application
}

João Paulo Barbosa Carvalho' ${ }^{1}$, Rosângela dos Santos Motta ${ }^{2}$

${ }^{1}$ Universidade de São Paulo, São Paulo - Brasil, joaopaulobc01@usp.br

2Universidade de São Paulo, São Paulo - Brasil, rosangela.motta@usp.br

\section{Recebido:}

13 de novembro de 2019

Aceito para publicação:

27 de agosto de 2020

Publicado:

30 de abril de 2021

Editor de área:

Jorge Barbosa Soares

\section{Palavras-chave:}

Agregados reciclados.

Dutos enterrados.

Módulo de resiliência.

Deformação permanente.

Pavimento.

\section{Keywords:}

Recycled aggregates.

Pipe backfilling.

Resilient modulus.

Permanent deformation.

Pavement.

DOI:10.14295/transportes.v29i1.2228

\section{RESUMO}

Este trabalho visa contribuir com a avaliação de agregados reciclados para uso em pavimentação, particularmente em reaterro de valas, possibilitando, assim, o reaproveitamento de materiais e buscando a melhoria da qualidade do pavimento reparado. Geralmente as especificações acerca do assunto indicam somente uma caracterização física básica dos materiais neste tipo de aplicação, deixando uma lacuna quanto ao seu comportamento mecânico. Para contribuir neste âmbito, o principal objetivo desta pesquisa é analisar o comportamento mecânico de agregados reciclados de resíduo de construção e demolição (RCD) e de reclaimed asphalt pavement (RAP) em laboratório. O RCD foi avaliado com e sem adição de cimento Portland, enquanto o RAP foi estudado na forma de mistura reciclada a frio com espuma de asfalto. Foi analisado o comportamento mecânico por meio de ensaios de módulo de resiliência e deformação permanente, sob carga repetida, além das evoluções de deformação permanente segundo a teoria do Shakedown. Adicionalmente, os resultados obtidos permitiram a proposição de seções-tipo com estes materiais reciclados em reaterro de valas. Os resultados mostram, de maneira geral, que o RCD estabilizado com cimento e o RAP podem ser aplicados como material de preenchimento de valas em diferentes tipos de tráfego, segundo as condições propostas.

\section{ABSTRACT}

This work aims to contribute to the evaluation of recycled aggregates for pavements, particularly in trench backfilling, thus enabling the reuse of materials and improving the quality of the pavement repaired. Generally, the specifications on this subject indicate only basic physical characterization of the materials in this type of application, leaving a gap in their mechanical behavior. To contribute to this field, the main objective of this research is to analyze the mechanical behavior of recycled construction and demolition waste (RCD) and reclaimed asphalt pavement (RAP) aggregates in the laboratory. RCD was evaluated with and without Portland cement, while RAP was studied as a cold recycled asphalt foam mix. The mechanical behavior was analyzed through resilient modulus and permanent deformation tests under repeated load, as well as permanent deformation evolutions according to the Shakedown theory. Additionally, the results obtained allowed the proposition of some pavement sections using these recycled materials in the trench backfilling. The results show, in general, that RCD with Portland cement and RAP can be applied as trench backfilling materials in different types of traffic, considering the conditions of this study. 


\section{INTRODUCÃO}

A necessidade de intervenções em pavimentos urbanos pelas concessionárias de saneamento básico e por companhias que utilizam redes subterrâneas para fornecimento de serviços (gás, energia, telefonia, fibra ótica, dentre outras) requer escavações em forma de trincheiras para reparar ou reinstalar novas tubulações e, posteriormente, aterrá-las.

As dificuldades encontradas no reaterro de valas geralmente se referem ao alívio de tensões do solo durante a abertura da vala, esgotamento d'água, espaço estreito de execução da compactação e compactação imprópria do material de preenchimento (Botelho, 1998; Ghataora et al., 2006; Godoy e Vasconcellos, 2013). Essas acarretam, além de riscos de acidentes e redução operacional da via, defeitos no pavimento, tais como falhas nas bordas de maneira adjacente ao corte da vala, buracos decorrentes de desagregação da camada de revestimento, recalque devido a acomodações plásticas e maior percolação de água nos materiais constituintes da vala.

Além disso, as adversidades mais recorrentes neste tipo de serviço (em que muitas vezes é utilizado o próprio material escavado) estão relacionadas à compactação dos materiais de preenchimento, excesso de umidade na trincheira, natureza mineralógica dos solos reutilizados e, no caso do município de São Paulo, os solos serem frequentemente de baixa capacidade de suporte e expansivos (SABESP, 2018).

Segundo o ranking de serviços em obras de reaterro de valas em pavimentos urbanos, as companhias de águas, gás, eletricidade e de trânsito da cidade de São Paulo foram notificadas várias vezes pela prefeitura municipal por apresentarem serviços com defeitos ou inacabadas. Considerando ainda que a quantidade de serviços de reaterro é significativa, estes problemas se tornam ainda mais evidentes. Para se ter uma ideia, no ano de 2017 a SABESP (Companhia de Saneamento Básico do Estado de São Paulo) realizou mais de 600 mil reposições de valas na região metropolitana da cidade de São Paulo, tendo ainda que efetuar muitas recorreções (quase 10.000 serviços refeitos naquele ano) (SABESP, 2018).

A utilização de materiais alternativos de qualidade, com adequada capacidade de suporte para esta finalidade pode ser uma alternativa para a melhoria da qualidade dos pavimentos reparados. Materiais como agregados reciclados de RCD e RAP vêm sendo empregados em obras de pavimentação, mas as especificações normalmente requerem somente uma caracterização física simplificada dos materiais, não compreendendo ensaios de comportamento mecânico que mostrariam sua condição mais próxima à de serviço.

Além do uso de materiais adequados no reaterro de valas, sua caracterização de comportamento mecânico ainda permite verificações estruturais na estrutura do pavimento, sob uma perspectiva mecanicista. 0 presente trabalho tem o objetivo apresentar um estudo laboratorial de agregados reciclados de RCD (com e sem cimento) e de RAP (na forma de mistura reciclada a frio com espuma de asfalto), do ponto de vista de comportamento mecânico, tendo em vista a aplicação destes materiais em reaterro de valas. Para tanto, foram realizados ensaios de módulo de resiliência e deformação permanente, onde o primeiro permitiu uma análise mecanicista e, consequentemente, a proposição de seções-tipo de estruturas de pavimentos com foco no reaterro de valas, enquanto o segundo permitiu compreender em detalhes a questão da deformação permanente, sob a ótica da teoria do Shakedown. Pretende-se, assim, contribuir para o maior entendimento do comportamento dos materiais reciclados analisados, indo além do que as especificações usualmente requerem (no caso uma caracterização física básica dos materiais). 


\section{REATERRO DE VALAS}

As intervenções nos pavimentos urbanos concentram-se na abertura de valas para a instalação e manutenção de tubos ou redes, seguida de restauração do próprio pavimento. As principais causas de falhas na recomposição das valas se dão quanto a: (i) métodos de construção que englobam técnicas de escavação e assentamento do material de preenchimento; (ii) materiais de preenchimento com baixa resistência com reutilização de materiais escavados saturados que afetam a compactação e resultam em expansão ou retração do reaterro; e (iii) local da vala, condicionando o tipo de material, condições de umidade e se o local está fora da canalização do tráfego (Schaefer et al., 2005; Widger e Zabolotnii, 2013; Andrade, 2016).

Ademais, sobre as adversidades frequentes com o material de preenchimento das valas, pode-se citar: (i) recalque inicial; (ii) recalque a longo prazo decorrente do carregamento do tráfego, colapso ou compactação não uniforme dos materiais ou recalque diferencial do solo da trincheira e do entorno e (iii) condições de umidade e congelamento, devido à presença de finos, permeabilidade dos materiais ou fenômeno de retração ou expansão dos materiais (GELB, 2002; Widger e Zabolotnii, 2013).

Após a instalação de dutos enterrados, a redistribuição de tensões no solo é fruto da interação entre o solo circundante e da estrutura, assim, um bom dimensionamento deve considerar as cargas móveis (tráfego), peso próprio do maciço de solo (tipo de solo, profundidade do tubo, empuxo e compactação) e a tubulação (tipo de tubo, tipo de instalação, rigidez do material) para minimizar os efeitos de arqueamento e deflexões (Bueno e Costa, 2012).

A SABESP e a Prefeitura do Município de São Paulo (PMSP) apresentam soluções de estruturas para a reposição do pavimento após a abertura de vala, em função do tipo de tráfego. A PMSP indica a aplicação de agregado reciclado de RCD como material de preenchimento de valas e/ou envoltória, com espessura variável dependendo do tráfego, onde o requisito preconizado é ter CBR $\geq 20 \%$, sob compactação realizada a 100\% de Proctor normal (PMSP, 2018).

Diferentes tipos de materiais têm sido estudados e aplicados como preenchimento em reaterro de valas. Dentre eles pode-se citar os granulares (Carrera et al., 2010), cinzas de carvão (Le et al., 2017), Controlled Low-Strength Materials - CLSMs (Ling et al., 2018) e pneu trituradoareia (Terzi et al., 2015). No Brasil, o material de preenchimento mais utilizado é o material escavado da própria vala. Entretanto, já há normativas vigentes no estado de São Paulo que possibilitam a utilização de outros materiais para o reaterro de valas, como a brita graduada simples (BGS), resíduo de construção e demolição (RCD), reclaimed asphalt pavement (RAP) e concreto magro, sendo a utilização de cada material em função do tráfego local (SABESP, 2016; PMSP, 2018).

De acordo com as normas ASTM D 2774 e D 2321 (ASTM, 2012 e 2018, respectivamente), os agregados reciclados de concreto podem ser utilizados como materiais de preenchimento e envoltória nas valas, sendo classificados como da Classe II, que engloba materiais de granulometria grossa (GW, GP, SW, SP) podendo conter até $12 \%$ de material fino (esta classe equivale a solos da categoria A1 e A3 da AASHTO, segundo ASTM, 2018). Agregados, detritos ou torrões congelados maiores que 3 polegadas $(76 \mathrm{~mm})$ de diâmetro devem ser removidos.

A Série 880 da Specification for Highway Works (SHW, 2016) classifica os agregados reciclados de concreto em três tipos - I, II e III (open graded), tratando de granulometria do material utilizado para preenchimento da vala, quantidade de finos (passantes na peneira 0,425 $\mathrm{mm}$ ) e conteúdo de materiais indesejáveis. Já a Melbourne Retail Water Agencies (MRWA) permite a 
utilização de agregados reciclados como material de preenchimento de reaterro de valas, especificando a granulometria e o grau de compactação de $95 \%$ para camadas entre $100 \mathrm{~mm}$ e 300 mm de espessura (MRWA, 2013).

\section{MATERIAIS E MÉTODOS}

Os materiais estudados nesta pesquisa para aplicação em reaterro de valas foram: (i) agregado reciclado de RCD (puro); (ii) agregado reciclado de RCD com cimento Portland e (iii) agregado reciclado de RAP com espuma de asfalto (mistura reciclada a frio).

0 agregado reciclado de RCD empregado nessa pesquisa foi oriundo da demolição de um muro de concreto, sendo classificado como RCD de concreto (ou cinza). Como será observado mais adiante, este RCD possuía originalmente graduação muito uniforme de dimensões da ordem de $32 \mathrm{~mm}$, que não se mostrou adequada nos primeiros ensaios mecânicos. Notou-se que a compactação em energia intermediária produzia quebra de agregados, gerando uma curva de graduação bem mais distribuída, com quantidade relativamente significativa de finos. Optouse, então, por avaliar este material sob "nova" curva granulométrica, agora com dimensão máxima de $19 \mathrm{~mm}$, a partir do reúso do material compactado (originalmente denominado "RCD-32").

As curvas granulométricas dos materiais estudados (principal base das especificações para RCD mencionadas no item anterior) foram obtidas por peneiramento (conforme MRWA, 2013; SHW, 2016), sendo o resultado apresentado na Figura 1.

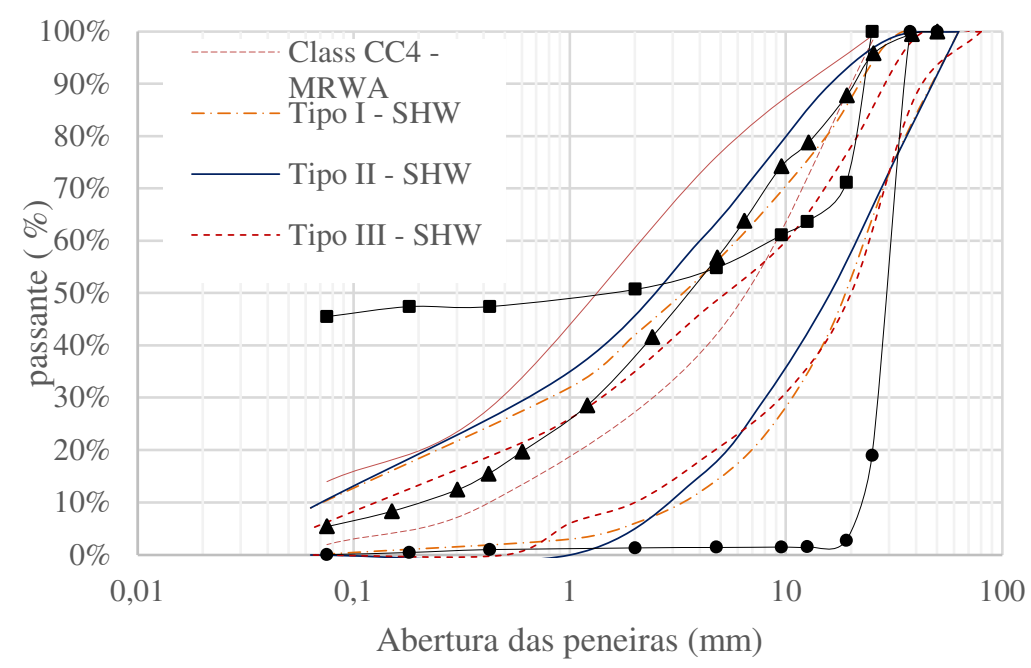

Figura 1. Curvas granulométricas dos agregados reciclados e comparação com as faixas de MRWA e SHW

Na Figura 1Figura 1 observa-se que o RCD não se enquadrou nas faixas especificadas por MRWA (2013) e SHW (2016), nem antes da compactação (curva original RCD-32) e nem depois de ter sofrido quebra neste processo (RCD-19). Esta quebra foi significativa, elevando a quantidade de finos. Mesmo fora das faixas especificadas, optou-se por prosseguir com as análises com estes materiais, tendo em vista as informações da literatura sobre sua quebra ser benéfica na compactação (Leite, 2007). Embora o RCD-32 tivesse curva granulométrica notavelmente uniforme e fora das faixas especificadas, seu percentual de finos atendia às normas ASTM D 2774 (2012) e D 2321 (2018), por ter menos de 12\% destes. Para comparação, o RAP também se enquadraria neste aspecto. 
Quanto às demais características do RCD, a Tabela 1 apresenta outros aspectos levantados a partir de ensaios laboratoriais nesta pesquisa.

O cimento Portland utilizado em mistura com o RCD era do tipo CP-II (comumente utilizado no Brasil), na proporção de 3\% em massa de agregado reciclado (porcentagem essa definida com base em trabalhos realizados por Motta, 2005 e Beja, 2014).

Tabela 1 - Características do RCD

\begin{tabular}{|c|c|c|}
\hline Parâmetro & Valor obtido & Especificação de ensaio \\
\hline Porcentagem de grãos cúbicos & $84 \%$ & \multirow{4}{*}{ NBR 5564 (ABNT, 2014) } \\
\hline Porcentagem de grãos alongados & $4 \%$ & \\
\hline Porcentagem de grãos lamelares & $12 \%$ & \\
\hline Porcentagem de grãos alongados lamelares & $0 \%$ & \\
\hline Abrasão Los Angeles (Graduação F) & $52 \%$ & DNER-ME 035 (DNER, 1998a) \\
\hline Absorção do agregado graúdo & $5,72 \%$ & DNER-ME 081 (DNER, 1998b) \\
\hline Massa específica aparente máxima* & $1,680 \mathrm{~g} / \mathrm{cm}^{3}$ & \multirow{2}{*}{ DNIT-ME 164 (DNIT, 2013) } \\
\hline Umidade ótima & $11,2 \%$ & \\
\hline
\end{tabular}

Já o agregado reciclado de RAP (mistura reciclada a frio com espuma de asfalto) foi oriundo de obras de pavimentação na BR-070 (Rodovia Ayrton Senna - SP), nas quais estava sendo aplicado este material. 0 material já usinado foi levado ao laboratório, sendo posteriormente compactado para os testes de comportamento mecânico. As características do RAP espumado são apresentadas na Tabela 2 . Cabe mencionar que a dosagem da mistura foi definida por meio dos ensaios de resistência à tração por compressão diametral e o método de ensaio de compactação do material teve como diretriz a norma DNIT-ME 164 (DNIT, 2013).

Tabela 2 - Características do RAP espumado

\begin{tabular}{ll}
\hline Tipo de ligante & CAP 50/70 \\
\hline Porcentagem de ligante asfáltico & $2,3 \%$ \\
Fíler ativo & Cal CH1 \\
Porcentagem de fíler ativo & $1 \%$ \\
Massa específica aparente máxima & $2,072 \mathrm{~g} / \mathrm{cm}^{3}$ \\
Umidade ótima & $10,2 \%$ \\
\hline *Compactação em energia Proctor modificada
\end{tabular}

Os ensaios para avaliação do comportamento mecânico compreenderam módulo de resiliência e deformação permanente em equipamento triaxial cíclico. Antes da realização destes, os corpos de prova compactados foram submetidos à cura de 7 dias. Sabe-se pela literatura que no caso do RCD (puro) podem ocorrer reações pozolânicas que geram cimentação (e, por consequência, levam a um aumento de rigidez), desde a compactação e podendo perdurar por meses. Por outro lado, como o cimento e o asfalto espumado tendem a apresentar o ganho significativo de rigidez logo nas primeiras idades, foi fixado o período de cura de 7 dias (Motta, 2005; Beja, 2014; Kuchiishi et al., 2019).

O ensaio de módulo de resiliência foi realizado segundo o método de ensaio DNIT ME 134 (DNIT, 2018) e, posteriormente, a análise dos resultados foi feita utilizando o modelo composto (em função da tensão confinante e desvio). Já o ensaio de deformação permanente foi conduzido a partir de uma análise prévia no programa ELSYM5 de uma estrutura de pavimento hipotética (em que seria instalada uma vala) para determinar a tensão vertical $\sigma_{1}$ a ser empregada no teste de laboratório. Para tanto, no ELSYM5 foram usados resultados de módulo de resiliência obtidos 
nos ensaios desta pesquisa, levando-se em conta uma tensão de confinamento $\sigma_{3}$ de 0,1 MPa e coeficientes de Poisson obtidos em literatura (Beja, 2014). As tensões verticais obtidas com os três materiais estudados (em kgf/ $\mathrm{cm}^{2}$ ) foram, 1,22 para o agregado reciclado de RCD, 2,03 para o RCD com cimento e 1,44 para o RAP espumado.

A partir daí, as análises de deformação permanente se basearam na relação de tensões $\sigma_{\mathrm{d}} / \sigma_{3}$ indicadas na Tabela 3. No caso do RAP, optou-se em realizar mais um par de tensões para analisar o seu comportamento frente ao colapso incremental mais adiante abordado, frente à teoria do Shakedown, uma vez que é considerado um material viscoelástico e quase não se deformou com as relações impostas.

Tabela 3 - Relações de tensões delimitadas para o ensaio de deformação permanente

\begin{tabular}{llll}
\hline Material & $\boldsymbol{\sigma}_{\boldsymbol{d}}\left(\mathbf{k g f} / \mathbf{c m}^{\mathbf{2}}\right)$ & $\boldsymbol{\sigma}_{\mathbf{3}}\left(\mathbf{k g f} / \mathbf{c m}^{\mathbf{2}}\right)$ & $\sigma_{\boldsymbol{d}} / \boldsymbol{\sigma}_{\mathbf{3}}$ \\
\hline \multirow{3}{*}{ RCD-19 } & 0,61 & 0,61 & 1 \\
& 0,90 & 0,30 & 3 \\
& 1,05 & 0,17 & 6 \\
\multirow{2}{*}{ RCD-19_3C (com cimento) } & 1,02 & 1,02 & 1 \\
& 1,53 & 0,51 & 3 \\
\multirow{3}{*}{ RAP } & 1,71 & 0,28 & 6 \\
& 0,70 & 0,70 & 1 \\
& 1,07 & 0,35 & 3 \\
& 1,22 & 0,20 & 6 \\
& 1,28 & 0,14 & 9 \\
\hline
\end{tabular}

Em seguida, os resultados de deformação permanente também foram avaliados à luz da teoria do Shakedown, como forma de se observar os níveis críticos de tensão nos materiais em questão (comportamento elástico, plástico, colapso) (Werkmeister et al., 2001).

\section{RESULTADOS E ANÁLISES}

Módulo de resiliência

A Figura 2 apresenta os resultados de módulo de resiliência dos materiais estudados, em função da tensão de confinamento $\sigma_{3}$ (por se tratarem de materiais com características granulares, sensíveis ao confinamento). Cabe mencionar que o resultado do agregado reciclado RCD-32 não foi apresentado, pois o material não resistiu às tensões de acomodação $\left(\sigma_{3} \quad 0,07 \mathrm{MPa}\right.$ e $\sigma_{d} 0,07$ $\mathrm{MPa}$ e $\sigma_{3} 0,07 \mathrm{MPa}$ e $\sigma_{d} 0,21 \mathrm{MPa}$ ), acarretando em deformação significativa do corpo de prova ainda nesta fase inicial do teste. Isto pode ter ocorrido devido ao grande número de vazios presentes no material e à falta de intertravamento das partículas. Esta condição fez com que se optasse por se trabalhar com RCD com diâmetro máximo inferior e distribuição granulométrica mais fina (RCD-19), obtida após compactação do material.

Tomando-se como referência o estudo de Motta (2005), que analisou o módulo de resiliência de RCD para ? 3 de aproximadamente $0,2 \mathrm{MPa}$ (centro de uma camada de base sob baixo volume de tráfego), nota-se que o RCD-19 apresentou módulo de resiliência dentro do esperado para uma camada de base granular de pavimento (PMSP, 2004b), que seria entre 100 e $500 \mathrm{MPa}$ (o comportamento granular deste material também ficou evidente pela inclinação da linha de tendência do gráfico da Figura 2). Este bom comportamento se deve ainda à compactação realizada em energia intermediária, como em estudo de Leite et al. (2011), que proporcionou quebra e maior intertravamento dos grãos, sendo benéfico para seu comportamento quanto à rigidez. 


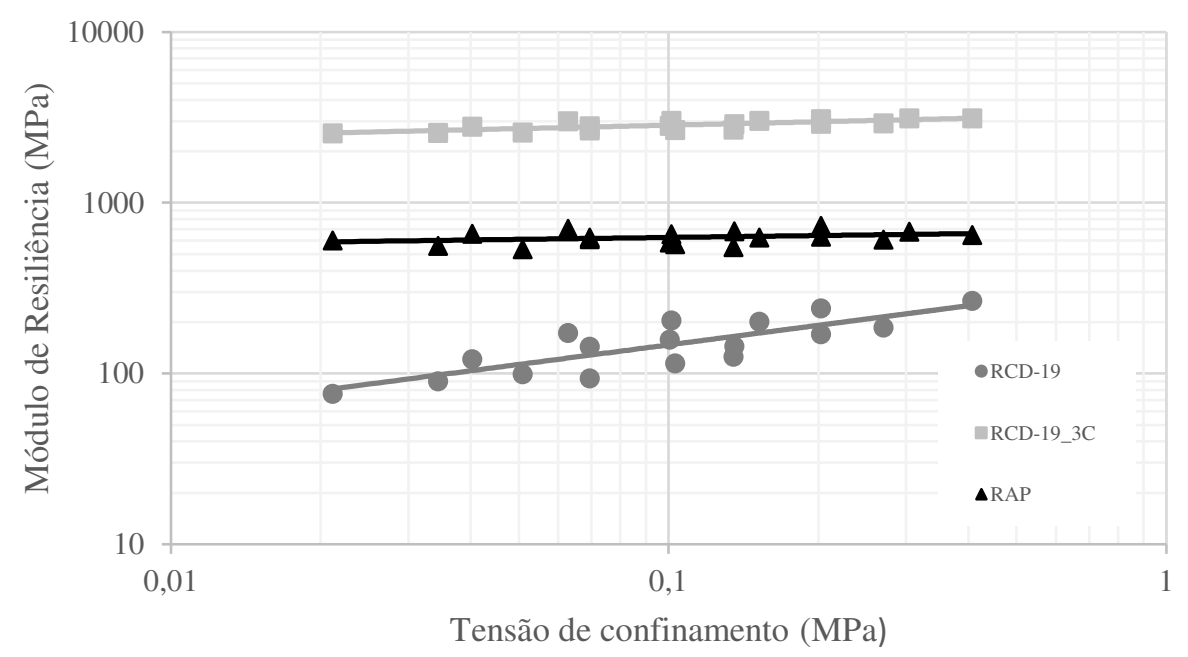

Figura 2. Módulo de resiliência dos agregados reciclados RCD e RAP

Quando foi adicionado 3\% de cimento ao RCD, verificou-se um incremento muito significativo do módulo de resiliência, como observado na Figura 2. Ou seja, é notória a ação do aglomerante hidráulico, causando um aumento na rigidez do material, logo elevando o módulo de resiliência. Este comportamento também foi obtido em outras pesquisas com agregado reciclado de RCD (Motta, 2005; Leite et al., 2011; Beja, 2014).

Além disso, notou-se que o RCD passou a apresentar comportamento menos dependente da tensão de confinamento com a adição de cimento, considerando que a linha de tendência da Figura 2 teve sua inclinação reduzida significativamente, o que seria de fato esperado para um material de tipo cimentado. Dado ainda este incremento notável (10 vezes ou mais, dependendo da tensão $\sigma_{3}$ tomada na linha de tendência), poderia se considerar a aplicação deste material em camada de preenchimento de valas de pavimento sujeito a condição de tráfego mais elevado, sob o ponto de vista de rigidez.

Quanto ao RAP, este apresentou valores de módulo de resiliência intermediários ao do RCD com e sem cimento, igualmente demonstrando resultados apropriados para seu uso como material de preenchimento de valas de pavimentos. Adicionalmente, assim como no caso do RCD com cimento, seu comportamento demonstrou ser pouco dependente da tensão de confinamento, não característico de materiais granulares. Deve-se ainda considerar que a energia de compactação modificada, que foi utilizada para o RAP, certamente contribuiu para seu bom comportamento quanto à rigidez.

Deve-se levar em conta ainda que os valores de módulo de resiliência desta pesquisa foram obtidos após cura somente de 7 dias e, como observado por outros autores da literatura (Motta, 2005; Beja, 2014; Kuchiishi et al., 2019), a tendência é de que a rigidez se eleve com mais tempo e, assim, maiores módulos de resiliência do que os encontrados neste estudo poderiam ser alcançados.

Os valores obtidos nos ensaios variaram de 491 a 845 MPa para o RAP espumado, de 78 a 256 MPa para o RCD-19 e de 2401 a 3982 MPa para o RCD-19_3C, dependendo da tensão de confinamento tomada na análise. Outras pesquisas encontraram valores similares: (i) Kuchiishi et al. (2019) de 620 a $1020 \mathrm{MPa}$ para o RAP espumado, (ii) Gómez (2011), entre 90 a $310 \mathrm{MPa}$ para o RCD puro e (iii) Beja (2014) entre 900 a 4500 MPa para o RCD estabilizado com 3\% de cimento. 


\section{Deformação permanente}

A Figura 3 apresenta os resultados de evolução da deformação permanente com o número de ciclos em multiestágios (relações de tensões da Tabela 4) para os três materiais estudado nesta pesquisa.

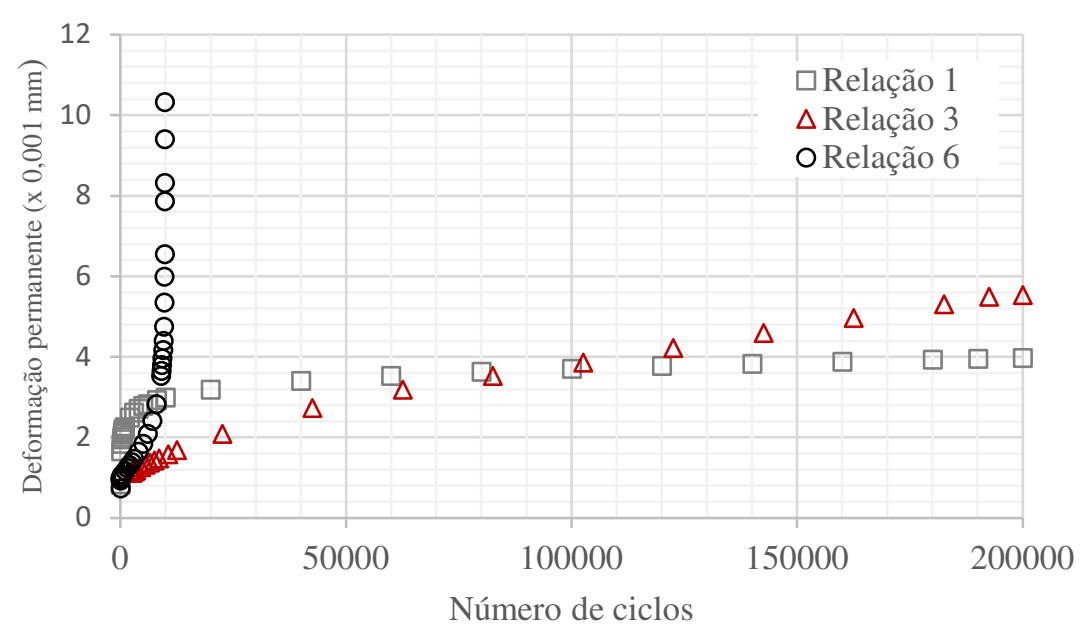

(a)

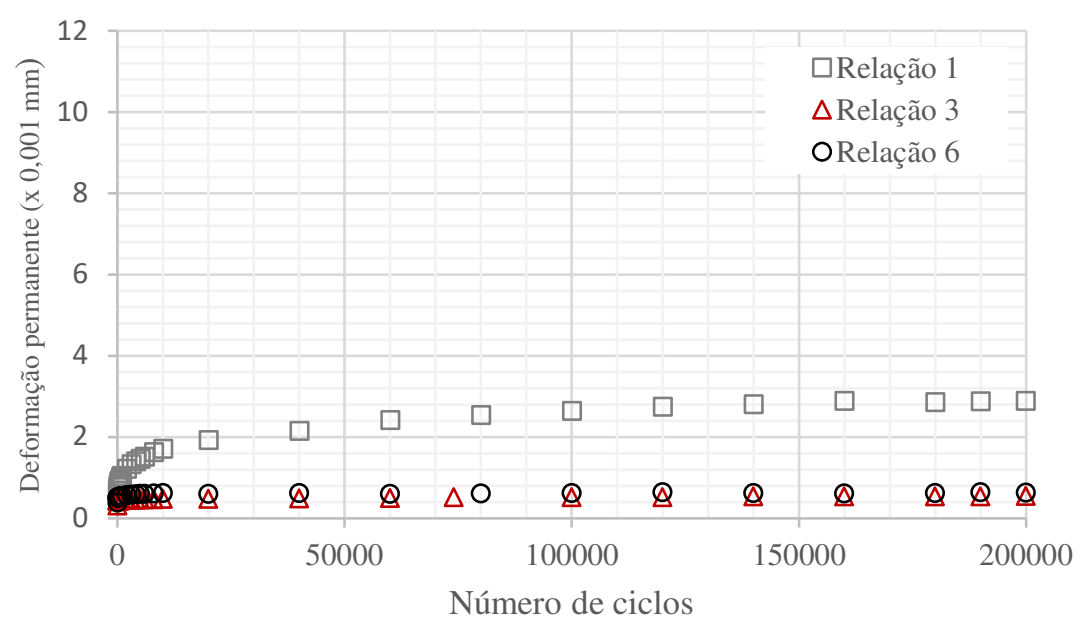

(b)

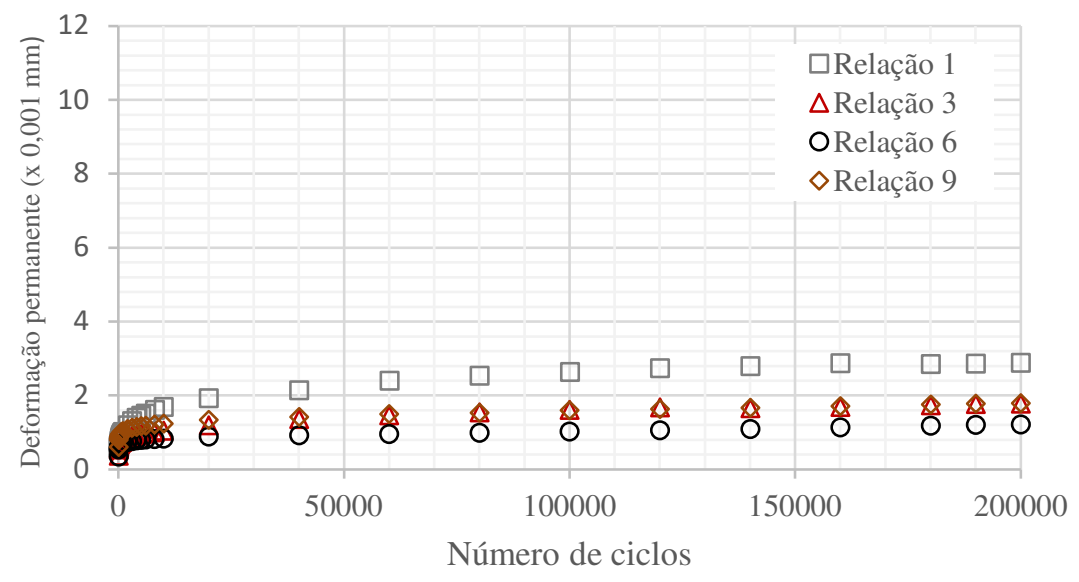

(c)

Figura 3. Evolução das deformações permanentes do RCD, RCD com $3 \%$ de cimento e RAP, respectivamente (a), (b) e (c) 
A partir dos resultados da Figura 3 a verifica-se que o RCD em relações de tensões mais baixas (1 e 3) apresenta uma certa deformação permanente inicial nos primeiros estágios, típica de materiais granulares. 0 comportamento de caráter granular também pode ser observado anteriormente no ensaio de módulo de resiliência. De maneira geral, após a acomodação inicial, este material apresentou uma tendência de estabilização da deformação permanente na relação 1 (considerando carregamento até 200.000 ciclos). Já ao se elevar a relação para 3, o material mostrou uma tendência contínua de acúmulo de deformação permanente ao longo dos ciclos analisados e, ao se elevar a combinação de tensões para a relação 6, o RCD apresentou colapso total, atingindo a deformação de $10 \%$ da altura do corpo de prova (critério de ruptura estabelecido para esta pesquisa, proposto por Malysz, 2004. Neste caso, que se trata de material granular, a deformação permanente será tanto maior quanto menor for a sua tensão confinante e maior a sua tensão desviadora, ou seja, a tensão de confinamento tende a inibir a deformação permanente e o acréscimo da tensão desvio tende a aumentar o aparecimento de deformações permanentes (Delongui et al., 2018).

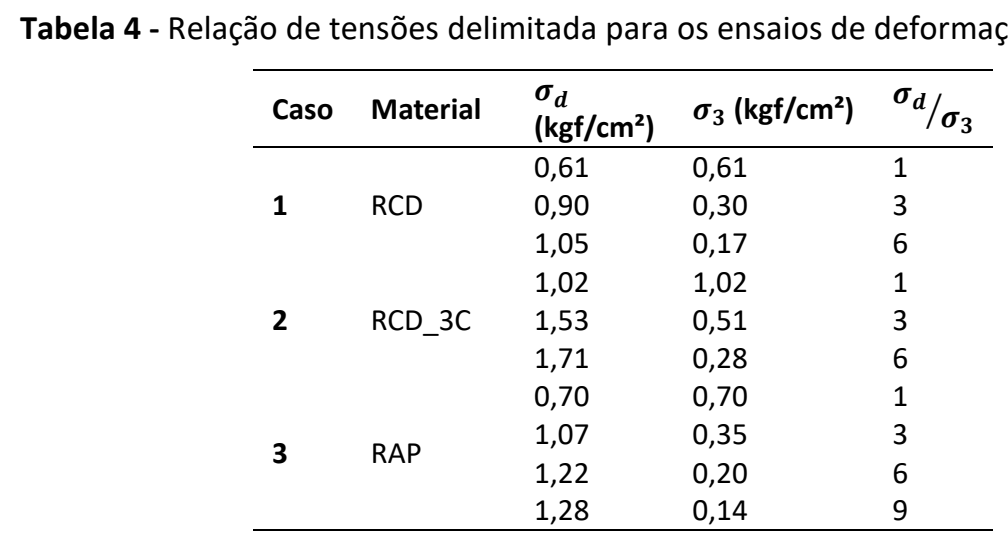

Já no caso do RCD com acréscimo de cimento (Figura 3b), observa-se que houve uma acomodação inicial, como no caso anterior, mas de maneira menos significativa, especialmente no caso das relações de tensões maiores (3 e 6). Verificou-se ainda que a adição de $3 \%$ de cimento foi benéfica no que se refere à deformação permanente, reduzindo-a em todas as relações de tensões, em comparação com o RCD (puro). Adicionalmente, nota-se que o nível de deformação permanente na relação de tensões mais baixa (1) foi mais elevado ao longo de todo o teste, no comparativo com as relações maiores (3 e 6). Não sendo este um material que apresentou comportamento típico de material puramente granular, como observado no ensaio de módulo de resiliência, o confinamento não foi preponderante na resistência à deformação permanente (Beja, 2014). Ademais, pode-se mencionar ainda que os baixos valores de deformação permanente observados nas relações mais elevadas (3 e 6) pode levar à consideração de uso deste material em preenchimento de valas com níveis de tráfego acima de tráfego leve, sob a ótica deste parâmetro.

Com relação ao material RAP, pela Figura 3c também se observa uma acomodação inicial, embora menor que no caso do RCD (típico granular). Do mesmo modo observado com o RCD com cimento, a menor relação de tensão levou a maior deformação permanente, cabendo as mesmas análises do caso anterior. 


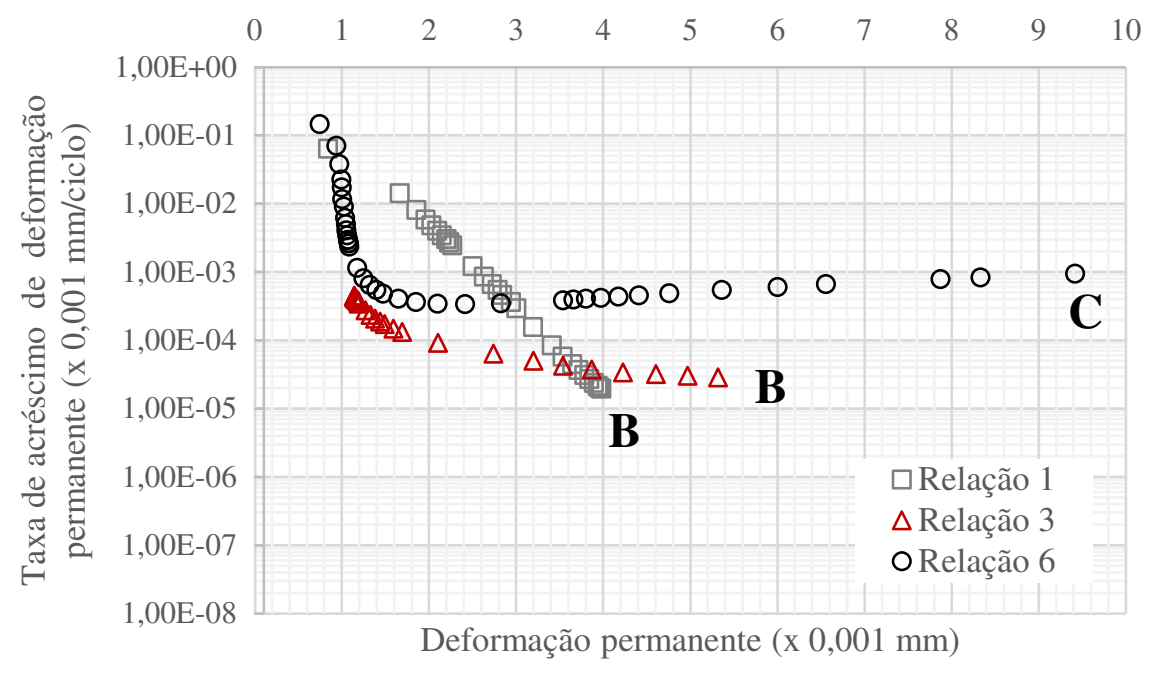

(a)

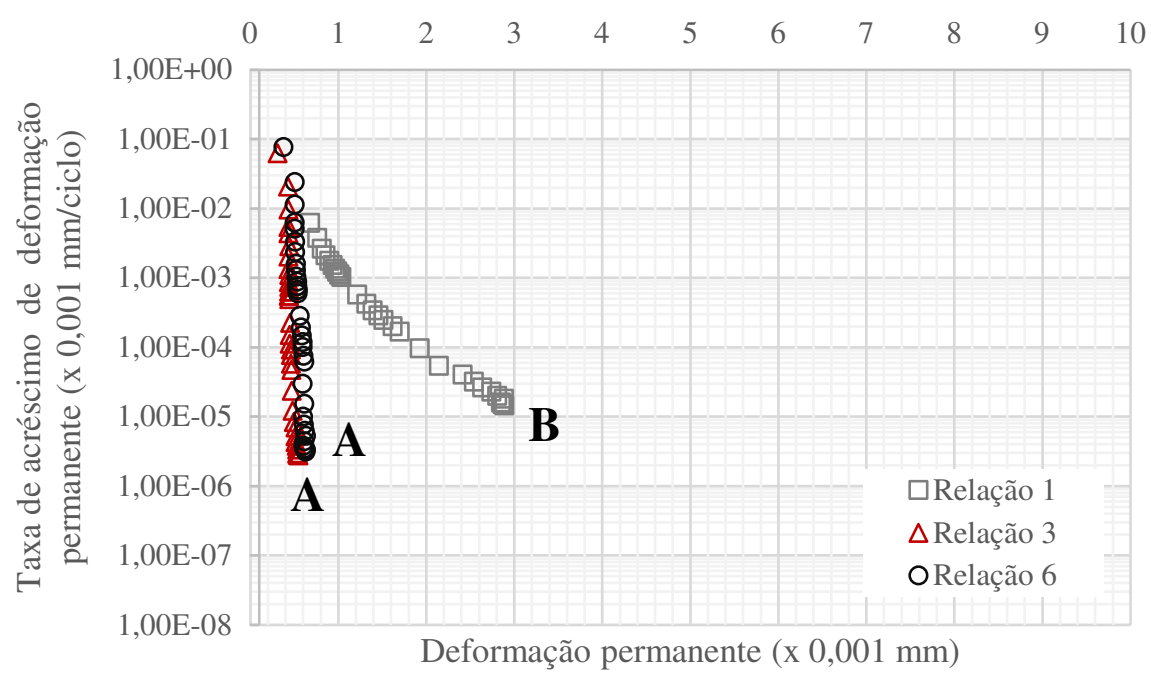

(b)

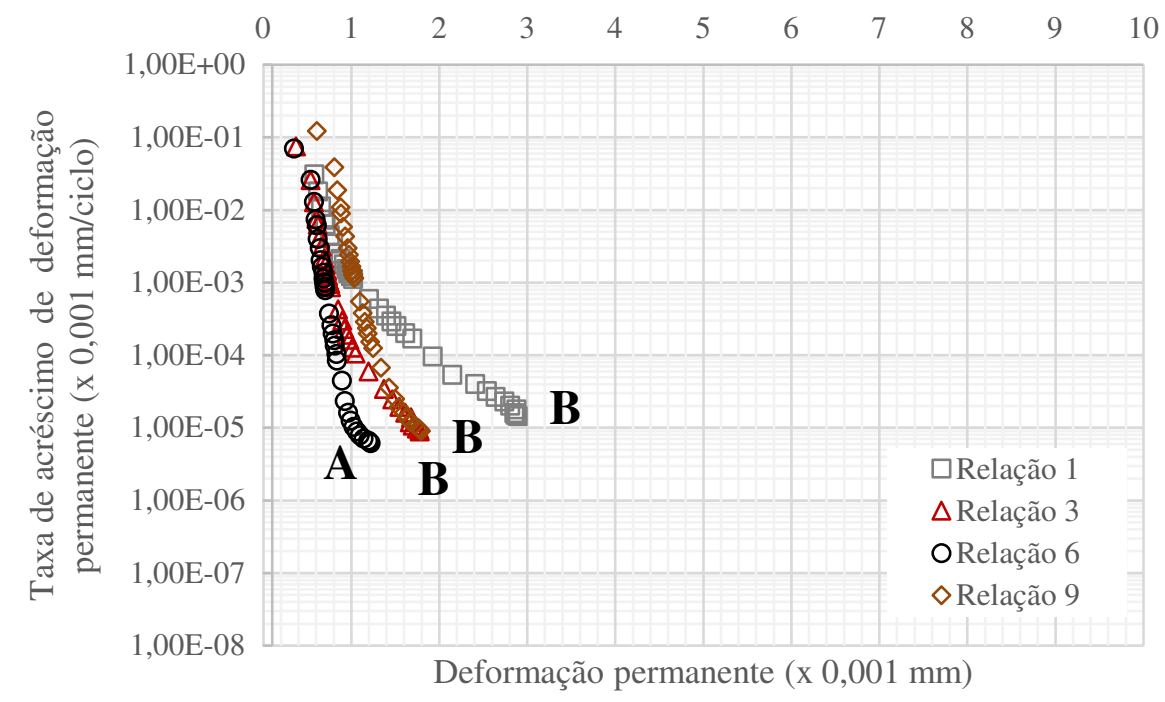

(c)

Figura 4. Análise segundo a teoria do shakedown do RCD, RCD com 3\% de cimento e RAP, respectivamente (a), (b) e (c) 
Por fim, nesta pesquisa foi feita também a avaliação da deformação permanente pela teoria do Shakedown. Neste caso, os resultados são dispostos de outra maneira, para se proceder com as análises. Da forma com que os resultados são plotados, pode-se categorizar os tipos de deformação, que pode seguir tendências de estabilização, intensificação de deformação por meio do incremento de ciclos ou a ruptura do material (Werkmeister et al., 2001). Para se obter esta análise os resultados foram plotados conforme proposto por Werkmeister et al. (2001), onde no eixo das ordenadas está representada a taxa de acréscimo de deformação permanente por número de ciclos e no eixo das abscissas encontra-se a deformação permanente. Os resultados são apresentados na Figura 4.

Baseado na Figura 4 é plausível afirmar que: (i) o Shakedown elástico (Faixa A) foi identificado nas relações 3 e 6 do RCD com cimento e na relação 6 do RAP, visto que as mesmas apresentam o formato retilíneo ou convexo, que configuram este comportamento; (ii) o colapso incremental (Faixa C) é representado por curvas com tendência a se tornarem horizontais, logo este comportamento foi identificado na relação 6 do RCD; (iii) o creep plástico ou Shakedown plástico (B) corresponde às outras curvas que não representam os comportamentos anteriores, ou seja, a maioria das curvas analisadas; (iv) na relação 3 do RCD, o arqueamento da curva aponta que a deformação permanente irá se acentuar ainda mais antes de chegar no colapso incremental, ou seja, o aumento no número de ciclos levará a curva a entrar na faixa C; e (v) o material RCD não apresentou faixa A e isto pode ser explicado devido ao mesmo apresentar cerca de $50 \%$ de finos em sua composição, o que acarretaria em maiores deformações permanentes para atingir a sua estabilização. Após análise dos resultados de deformação permanente frente a teoria do Shakedown, foram intitulados os seguintes limites para os materiais estudados nesta pesquisa (Tabela 5):

Tabela 5 - Limites do Shakedown para os materiais estudados

\begin{tabular}{lll}
\hline Caso & Material & Limites do Shakedown observados \\
\hline 1 & RCD & $\begin{array}{l}\text { Shakedown plástico }\left(1 \leq \sigma_{d} / \sigma_{3}<3\right) \\
\text { Colapso incremental }\left(\sigma_{d} / \sigma_{3}=6\right)\end{array}$ \\
$\mathbf{2}$ & RCD_3C & $\begin{array}{l}\text { Shakedown elástico }\left(1 \leq \sigma_{d} / \sigma_{3}<3\right) \\
\text { Shakedown plástico }\left(\sigma_{d} / \sigma_{3}>6\right)\end{array}$ \\
3 & RAP & $\begin{array}{l}\text { Shakedown elástico }\left(\sigma_{d} / \sigma_{3}=6\right) \\
\text { Shakedown plástico }\left(1<\sigma_{d} / \sigma_{3}>3 ; \sigma_{d} / \sigma_{3}>9\right)\end{array}$ \\
\hline
\end{tabular}

\section{PROPOSIÇÃO DE SEÇÕES-TIPO}

Com base nos resultados dos ensaios laboratoriais foram propostas sete seções-tipo (ST) de estrutura de pavimentos com os materiais estudados para a aplicação em reaterro de valas urbanas (Tabela 5), que foram obtidas com auxílio do programa ELYSM 5. Para tanto, utilizou-se uma carga de $2050 \mathrm{kgf}$, com o tráfego e a análise mecanicista estipulados pela PMSP para pavimentos urbanos (PMSP, 2004a; PMSP, 2004b; PMSP, 2018). É importante ressaltar que a camada de envoltória da tubulação tem espessura variável, logo é função de cada projeto e suas particularidades (tipo de tráfego, tipo de tubulação, tipo de solo remanescente, entorno das paredes da vala, dentre outros aspectos).

No caso do RCD sem cimento não foi considerada nenhuma estrutura para tráfego de médio e pesado, devido à análise do Shakedown mostrar um comportamento colapsível acima de 
400000 ciclos, mesmo que o programa Elsym5 indique a possibilidade de utilizar espessuras maiores para vencer as deformações e tensões nas camadas. A estrutura proposta para o RCD está em consonância à proposição indicada na IR-01 (PMSP, 2018) para tráfego leve e em contrapartida está com espessura maior de revestimento e menor de material de base quando comparada com a NTS 164 (SABESP, 2016). Por outro lado, as estruturas que utilizam base de RAP e RCD com cimento apresentaram espessuras menores aos apresentados na IR-01 (PMSP, 2018) e NTS 164 (SABESP, 2016), possivelmente função do módulo de resiliência mais elevado (maior rigidez). A análise das deformações permanentes pela teoria do Shakedown mostrou que o material tem resistência a deformações advindas do tráfego. Por fim, a análise mecanicista mostrou que todas as verificações tiveram valores abaixo do admissível pelos modelos utilizados (2004b). De forma a assegurar a tubulação é desejável que aplique-se $15 \mathrm{~cm}$ de areia compactada na energia normal na parte acima do topo da tubulação e $5 \mathrm{~cm}$ de areia compactada na energia normal na parte abaixo da base da tubulação (SABESP, 2016), com a finalidade de proteção da tubulação das demais compactações acima da camada de envoltória.

Tabela 6 - Seções-tipo propostas para os agregados reciclados desta pesquisa

\begin{tabular}{|c|c|c|c|c|c|}
\hline \multicolumn{6}{|c|}{ TRÁFEGO LEVE - $\mathrm{N}=10^{5}$} \\
\hline ST1 & Espessura (cm) & ST2 & Espessura (cm) & ST3 & Espessura $(\mathrm{cm})$ \\
\hline CAUQ & 8 & CAUQ & 3,5 & CAUQ & 3,5 \\
\hline $\mathrm{RCD}$ & 10 & RCD_3C & 7 & RAP & 10 \\
\hline Areia & $\infty$ & Areia & $\infty$ & Areia & $\infty$ \\
\hline \multicolumn{6}{|c|}{ TRÁFEGO MÉDIO - $\mathrm{N}=5 \times 10^{5}$} \\
\hline & ST4 & Espessura (cm) & ST5 & Espessura (cm) & \\
\hline & CAUQ & 3,5 & CAUQ & 5 & \\
\hline & RCD_3C & 10 & RAP & 15 & \\
\hline & Arēia & $\infty$ & Areia & $\infty$ & \\
\hline \multicolumn{6}{|c|}{ TRÁFEGO PESADO - $\mathrm{N}=2 \times 10^{7}$} \\
\hline & ST6 & Espessura (cm) & ST7 & Espessura $(\mathrm{cm})$ & \\
\hline & CAUQ & 5 & CAUQ & 8 & \\
\hline & RCD_3C & 15 & RAP & 25 & \\
\hline & Areia & $\infty$ & Areia & $\infty$ & \\
\hline
\end{tabular}

Foi proposto na Tabela 6 um estudo de deflexões recuperáveis das diversas camadas das estruturas do pavimento, como ferramenta adicional no controle tecnológico de obra. Portanto, esta indicação serviria como balizador para uma aceitação ou não dos serviços (controle de qualidade).

Tabela 7 - Deflexão do controle deflectométrico - medidas em (x10-2 mm)

\begin{tabular}{llllllll}
\hline CAMADA & ST1 & ST2 & ST3 & ST4 & ST5 & ST6 & ST7 \\
\hline CAUQ & 57 & 60 & 65 & 51 & 54 & 40 & 41 \\
RCD & 104 & - & - & - & - & - & - \\
RCD_3C & - & 76 & - & 63 & - & 49 & - \\
RAP & - & - & 81 & - & 68 & - & 55 \\
Areia & 120 & 120 & 120 & 120 & 120 & 120 & 120 \\
\hline
\end{tabular}

\section{CONCLUSÕES}

De maneira geral, os resultados de módulo de resiliência demonstraram que os agregados reciclados de RCD com cimento e os agregados reciclados de RAP com espuma de asfalto podem alcançar 
valores compatíveis com estruturas de pavimentos em que seriam feitas obras de valas. Já com relação à deformação permanente, verificou-se que o agregado reciclado de RCD pode ser capaz de suportála, mas não em relações de tensões de maiores valores. Neste caso, a incorporação de cimento foi extremamente benéfica, trazendo a deformação permanente para patamar mais baixo, inclusive em relações de tensões mais elevadas. Isto reforça o resultado encontrado no ensaio de módulo de resiliência, onde a adição do ligante hidráulico pode sugerir o uso deste material em pavimentos sujeitos a tráfego mais elevado.

A análise do comportamento considerando a teoria do Shakedown apontou a maior susceptibilidade do agregado reciclado de RCD ao Shakedown, chegando a colapso incremental. Porém, com a adição de cimento Portland, essa susceptibilidade foi reduzida, melhorando significativamente a resposta à deformação permanente.

Quanto às seções-tipo propostas nesta pesquisa, com exceção do RCD sem cimento, os demais materiais (RAP e RCD com cimento) apresentaram estruturas mais delgadas daquelas propostas pelos normativos da PMSP e da SABESP. O RCD sem cimento se mostrou apto para a sua aplicação em pavimentos do tipo de tráfego leve; neste caso, verifica-se a importância da caracterização do comportamento mecânico dos materiais de pavimentação em laboratório, somada à análise mecanicista, para conferir a aptidão do uso de um determinado material.

\section{AGRADECIMENTOS}

Os autores agradecem a doação de materiais pela Prefeitura do Campus USP da Capital e pela empresa FREMIX e ao CNPq pelo financiamento da bolsa do primeiro autor.

\section{REFERÊNCIAS BIBLIOGRÁFICAS}

Andrade, L. R. (2016) Boas práticas para obras de reaterro de valas e recomposição de pavimentos. Dissertação (Mestrado em Habitação: Planejamento e Tecnologia). Programa de Pós-Graduação IPT, Instituto de Pesquisas Tecnológicas do Estado de São Paulo. São Paulo. Disponível em: < https://www.ipt.br/pos_graduacao_ipt/solucoes/dissertacoes/836-boas_praticas_para_obras_de_reaterro_de_valas_e_recomposicao_de_pavimentos.htm\#: :text=Entre\%20as\%20boas\%20pr\%C3\%A1ticas\%20abordadas,materiais\%20apropriados\%20para\%20o\%20uso> (acesso em 15/07/2018).

American Society for Testing and Materials (ASTM) (2012) ASTM D 2774: standard practice for underground installation of thermoplastic pressure piping.

American Society for Testing and Materials (ASTM) (2018) ASTM D 2321: standard practice for underground installation of thermoplastic pipe for sewers and other gravity-flow applications.

Associação Brasileira de Normas Técnicas (ABNT) (2014) ABNT NBR 5564: Via férrea - Lastro ferroviário - requisitos e métodos de ensaio.

Beja, I. A. (2014) Agregado reciclado de construção e demolição com adição de aglomerantes hidráulicos como sub-base de pavimentos. Dissertação (Mestrado), Programa de Pós-Graduação em Engenharia de Transportes, Escola Politécnica da Universidade de São Paulo. São Paulo. Disponível em: <https://www.teses.usp.br/teses/disponiveis/3/3138/tde-16102014151237/pt-br.php> (acesso em 21/07/2018).

Botelho, M. H. C. (1998). Águas de chuva: engenharia das águas pluviais nas cidades. $2^{\circ}$ ed. Edgard Blucher, São Paulo.

Bueno, B. e Y. Costa. (2012) Dutos enterrados: aspectos geotécnicos. 2oe ed. Oficina de Textos, São Paulo.

Carrera, A.; J. Grenfell; A. Dawson e J. Proctor. (2010). On site recycling of trench arisings for pavement reinstatement. Disponível em: < http://www.claisse.info/2010\%20papers/k6.pdf> (acesso em 09/08/2018).

Companhia de Saneamento Básico do Estado de São Paulo (2016) NTS 164: Ramal predial de água - diâmetro externo nominal de 20 e $32 \mathrm{~mm}$ em polietileno. São Paulo.

Companhia de Saneamento Básico do Estado de São Paulo (2018) Termo de referência. São Paulo.

Delongui, L.; M. Matuella; W. P. Núñez; W. Fedrigo; L. C. P. Silva Filho e J. A. P. Ceratti. (2018) Construction and demolition waste parameters for rational pavement. Construction and Building Materials. v. 168, p. 105-112. DOI: 10.1016/j.conbuildmat.2018.02.086

Departamento Nacional de Estradas de Rodagem (DNER). (1998a) DNER ME 35: agregados - determinação da abrasão "Los Angeles".

Departamento Nacional de Estradas de Rodagem (DNER). (1998b) DNER ME 81: agregados - determinação da absorção e da densidade de agregado graúdo.

Departamento Nacional de Infraestrutura de Transportes (DNIT). (2013) DNIT ME 164: Solos - compactação utilizando amostras não trabalhadas - método de ensaio. 
Departamento Nacional de Infraestrutura de Transportes (DNIT). (2018) DNIT ME 134 Pavimentação - Solos - Determinação do módulo de resiliência - método de ensaio.

Department of the Environment and Local Government (GELB) (2002) Guidelines for the opening, backfilling and reinstatement of trenches in public roads. Ireland. Disponível em: < https://www.roadex.org/wp-content/uploads/2014/01/TrenchGuidelines.pdf> (acesso em 02/08/2018).

Ghataora, G. S.; I. Alobaidi; E. Faragher e S. Grant. (2006). Use of recycled aggregates for cementitious backfill. Waste and Resource Management. v. 159, p. 2. DOI: 10.1680/warm.2006.159.1.23.

Godoy e Vasconcellos, J. L. (2013). Valas: abertura, escoramento provisório e esgotamento d'água. Ed. Baraúna. São Paulo.

Gómez, A. M. J. (2011) Estudo experimental de um resíduo de construção e demolição (RCD) para utilização em pavimentação. Dissertação (Mestrado). Programa de Pós-Graduação em Geotecnia, Universidade de Brasília. Brasília. Disponível em: < https://repositorio.unb.br/handle/10482/9514 > (acesso em 18/07/2018).

Kuchiishi, A. K.; C. C. S. Antão; K. L. Vasconcelos; J. J. Pires; O. M. O. Araújo; L. L. B. Bernucci e R. T Lopes. (2019) Investigation of the matric suction role on the curing mechanism of foamed asphalt stabilized mixtures. Road Materials and Pavement Design. v. 20, p. 365-389. DOI: 10.1080/14680629.2019.1589558.

Le, H. M. T.; D. W. Park; J. W. Seo e W. J. Seo. (2017) Trench backfill material using plant coal ash. In: World of Coal Ash. Anais., p. 7. Disponível em: <http://www.flyash.info/2017/013p-Park-woca2017p.pdf> (acesso em 11/08/2018).

Leite, F. C. (2007) Comportamento mecânico de agregado reciclado de resíduo sólido da construção civil em camadas de base e sub-base de pavimentos. Dissertação (Mestrado), Programa de Pós-Graduação em Engenharia de Transportes, Universidade de São Paulo. São Paulo. Disponível em: <https://www.teses.usp.br/teses/disponiveis/3/3138/tde-09012008162141/pt-br.php> (acesso em 15/07/2018).

Leite, F. C.; R. S. Motta; K. L. Vasconcelos e L. L. B. Bernucci. (2011) Laboratory evaluation of recycled construction and demolition waste for pavements. Construction and Building Materials. v. 25, p. 2972-2979. DOI: 10.1016/j.conbuildmat.2010.11.105.

Ling, T. C.; S. K. Kaliyavaradhan e C. S. Poon. (2018) Global perspective on application of controlled low strength material (CLSM) for trench backfilling - an overview. Construction and Building Materials. v. 158. P. 535-548. DOI: 10.1016/j.conbuildmat.2017.10.050.

Malysz, R. (2004) Desenvolvimento de um equipamento triaxial de grande porte para avaliação de agregados utilizados como camada de pavimentos. Tese (Doutorado). Programa de Pós-Graduação em Engenharia Civil, Universidade Federal do Rio Grande do Sul. Porto Alegre. Disponível em: < https://www.lume.ufrgs.br/handle/10183/19046> (acesso em 23/07/2018).

Melbourne Retail Water Agencies (MRWA) (2013) Specification 04-03.2: Backfill Specification. Melbourne. Disponível em: <https://www.chw.net.au/mvc/K12WebApi/media/Development/Land\%20Development/Products,\%20Standards\%20and\%20Codes/MRWA_Backfill_Specification_04-03-2.pdf?ext=.pdf> (acesso em 09/07/2018).

Motta, R. S. (2005) Estudo laboratorial de agregado reciclado de resíduo sólido da construção civil para aplicação em pavimentação de baixo volume de tráfego. Dissertação (Mestrado), Programa de Pós-Graduação em Engenharia de Transportes, Universidade de São Paulo. São Paulo. Disponível em: < https://teses.usp.br/teses/disponiveis/3/3138/tde-19072006114729/pt-br.php > (acesso em 15/07/2018).

Prefeitura do Município de São Paulo (PMSP) (2004a) IP 04: Classificação das Vias. São Paulo.

Prefeitura do Município de São Paulo (PMSP) (2004b) IP 08: Análise mecanicista à fadiga de estruturas de pavimento. São Paulo.

Prefeitura do Município de São Paulo (2018) PMSP IR 01: instrução de reparação de pavimentos flexíveis danificados por abertura de valas. São Paulo.

Schaefer, V.; M. Suleiman; D. Write; C. Swan e K. Jensen. (2005). Utility cut repair techniques: investigation of improved cut repair techniques to reduce settlement in repaired areas. Iowa State University, Iowa. Disponível em: < https://intrans.iastate.edu/research/completed/utility-cut-repair-techniques-to-reduce-settlement-in-repaired-areas-phase-2-tr-566/> (acesso em 07/07/2018).

Specification for Highway Works (SHW) (2016) Series 800: road pavements - unbound, cement and other hydraulically bound mixtures. England. Disponível em: <https://www.standardsforhighways.co.uk/ha/standards/mchw/vol1/pdfs/MCHW\%20800.pdf> (acesso em 30/06/2018).

Terzi, N. U.; C. Erenson e M. E. Selçulk. (2015) Geotechnical properties of tire sand mixtures as backfill for buried pipe installations. Geomechanics and Engineering. v. 9. n. 4. p. 19. DOI: 10.12989/gae.2015.9.4.000.

Werkmeister, S.; A. Dawson e F. Wellner. (2001) Permanent deformation behavior of granular materials and the Shakedown concept. Transportation Research Record n. 1757, p. 75 -81. DOI: 10.3141/1757-09.

Widger, A. e E. Zabolotnii. (2013). Guidelines for improved trench reinstatement programs and work practices. Communities of Tomorrow Leveraged Municipal Innovation Fund. 